\title{
Improved Framingham Risk Scores of Patients with Type 2 Diabetes Mellitus in the Beijing Community: A 10-Year Prospective Study of the Effects of Multifactorial Interventions on Cardiovascular Risk Factors (The Beijing Communities Diabetes Study 22)
}

\author{
Xue-Lian Zhang · Gang Wan · Ming-Xia Yuan · Guang-Ran Yang · Han-Jing Fu • \\ Liang-Xiang Zhu · Rong-Rong Xie · Yu-Jie Lv · Jian-Dong Zhang · Yu-Ling Li · Qin-Fang Dai • \\ Yu Ji · Rury R. Holman · Shen-Yuan Yuan on behalf of Beijing Communities Diabetes Study Group
}

Received: December 13, 2019 / Published online: February 21, 2020

(c) The Author(s) 2020

\section{ABSTRACT}

Introduction: To date, research is lacking on the development of a cardiovascular disease (CVD) risk assessment tool for people with diabetes mellitus, in general, and for Chinese patients with diabetes in particular. We have

Xue-Lian Zhang and Gang Wan are co-first authors.

The members of Beijing Communities Diabetes Study Group are listed in the Electronic Supplementary Material

Enhanced Digital Features To view enhanced digital features for this article go to https://doi.org/10.6084/ m9.figshare.11799291.

Electronic supplementary material The online version of this article (https://doi.org/10.1007/s13300020-00782-5) contains supplementary material, which is available to authorized users.

X.-L. Zhang · M.-X. Yuan · G.-R. Yang · H.-J. Fu · L.-X. Zhu - R.-R. Xie - S.-Y. Yuan $(\bowtie)$

Department of Endocrinology, Beijing Tongren Hospital, Capital Medical University, Beijing, China e-mail: fulvic@126.com

G. Wan

Medical records and Statistics Department, Beijing Ditan Hospital, Capital Medical University, Beijing, China explored CVD risk assessment tools for Chinese patients with diabetes. Here, we report our investigation of cardiovascular risk assessment using the improved Framingham Risk Score (IFRS) in patients with type 2 diabetes mellitus (T2DM) in Beijing communities.

Methods: A total of 3232 patients with T2DM attending Beijing community health centers were enrolled in this study. FRS were used to predict CVD risk in all patients at baseline using the following risk scores for glycated hemoglobin (HbA1c) categories: $0=$ HbA1c $\leq 7.0 \%$; 1 $=7.0 \%<\mathrm{HbA} 1 \mathrm{c} \leq 7.9 \% ; \quad 2=8.0 \%<\mathrm{HbA} 1 \mathrm{c}$ $\leq 8.9 \%$; and $3=\mathrm{HbA} 1 \mathrm{c}>9.0 \%$. The I-FRS was use to stratify all patients into low (I-FRS < 10\%), medium (I-FRS 10-20\%), and high (I-FRS $>20 \%$ ) FRS strata. All treatments administered in the Beijing Communities Diabetes Study were in accordance with national guidelines for T2DM in China, and patients

Y.-J. Lv

Cuigezhuang Community Health Service Center, Beijing, China

J.-D. Zhang Jinsong Community Health Service Center, Beijing, China

Y.-L. Li

Xinjiekou Community Health Service Center, Beijing, China 
regularly attended clinical consultations with professors in endocrinology, who were experts in their respective speciality, from top tier hospitals. After 10 years, patients were followed-up to assess the long-term effects of the multifactorial interventions. Statistical analysis was performed using SAS ${ }^{\circledR}$ software (SAS Institute, Inc., Cary, NC, USA).

Results: The receiver operating characteristic curve of the I-FRS showed significant prediction accuracy for the actual incidence of CVD events. At baseline, subjects in the high FRS stratum for diabetes were more prone to be elderly and to have a longer duration of T2DM, higher systolic blood pressure, and higher lipid profiles. Subjects in the medium and high FRS strata had a higher incidence of CVD events than those in the no-complications group (DM group with no blood pressure issues) $(P<0.001)$. The 10 -year hazard ratios for CVD events in diabetic patients with I-FRS score $>$ $20 \%$ was 12.5 -fold higher than that of patients with I-FRS score $<10 \%$. Multifactorial intervention significantly reduced the I-FRS of the three FRS strata in patients with T2DM. The post-intervention I-FRS for the hypertension and CVD groups of patients were significantly lower than the respective baseline I-FRS. Cox multivariate analyses revealed that patients in the medium and high FRS strata had higher incidences of endpoint events than those in the low FRS stratum.

Conclusions: The I-FRS plays an important role in predicting CVD in patients with T2DM. Multifactorial interventions for CVD risk factors over 10-year follow-up lowered the estimated 10-year risk for CVD events in persons with diabetes. We suggest the use of the I-FRS score

\section{Q.-F. Dai}

Yuetan Community Health Service Center of Fuxing Hospital, Capital Medical University, Beijing, China

Y. Ji

Department of Endocrinology, Beijing Aerospace

General Hospital, Beijing, China

R. R. Holman $(\bowtie)$

Diabetes Trials Unit, Oxford Centre for Diabetes, Endocrinology and Metabolism, University of Oxford, Oxford, UK

e-mail: rury.holman@dtu.ox.ac.uk to stratify a patient's risk of CVD when analyzing the efficacy of diabetes management. Aggressive risk reduction should be focused on those individuals with a high I-FRS score.

Trial Registration: ChiCTR-TRC-13003978 and ChiCTR-OOC-15006090.

Keywords: Cardiovascular disease; Framingham Risk Score; Hypertension; The Beijing Communities Diabetes Study; Type 2 diabetes mellitus (T2DM); UKPDS score

\section{Key Summary Points}

Our research focuses on the joint management model of community hospitals and a top tier hospital as applied to a large sample of patients with type 2 diabetes mellitus (T2DM) followed-up for 10 years.

Research has lagged on the development a cardiovascular disease (CVD) risk assessment tool for people with T2DM, and what few tools are currently available have often been copied or borrowed from other risk assessment models. It is therefore necessary to explore CVD risk assessment tools for Chinese patients with T2DM.

The aim of the Beijing Communities Diabetes Study was to investigate whether multifactorial interventions have a longterm effect on the risk of diabetes-related macro-vascular complications in patients with T2DM living in urban communities of Beijing. It is the first of its kind ever undertaken in China.

To date, with the major exception of the UK Prospective Diabetes Study, there have been few successful long-term prospective studies of diabetic management in a large sample of patients worldwide. Our results show that multifactorial interventions for CVD risk factors over 10-year lowered the estimated 10-year risk for CVD events in patients with diabetes. 
We suggest the use of the improved Framingham Risk Score (FRS) to stratify a patient's CVD risk when analyzing the efficacy of management. Aggressive risk reduction should be focused on those individuals with had a strongly improved (FRS) score.

\section{INTRODUCTION}

Diabetes is a prime risk factor for coronary heart disease [1]. Over the past three decades, the number of people with diabetes mellitus has more than doubled globally, making it one of the most important public health challenges worldwide [2]. The International Diabetes Federation estimated that there were 150 million people aged $\geq 20$ years living with diabetes in 2000 and that by 2025 this number will have risen to 300 million. Diabetes elevates the risk for cardiovascular disease (CVD) complications, particularly atherosclerotic vascular disease. CV events remain the major cause of death for those with diabetes, accounting for about $70 \%$ of all deaths in this patient population [3].

Epidemiological studies of CVD in the diabetes patient population are important in terms of being able to estimate both the magnitude and nature of the condition, to identify the risk factors for CVD in diabetic subjects, and to develop preventive measures. It should also be noted that relatively longer follow-ups may be necessary to see the benefit of diabetes therapies, as has been reported for the significant reduction of $\mathrm{CV}$ risk (including myocardial infarction) in patients with type 2 diabetes mellitus (T2DM) in the UK Prospective Diabetes Study (UKPDS) 10-year follow-up study [4]. To date, with the exception of the UKPDS, there have been few successful long-term prospective studies of diabetic management in a large sample of patients worldwide.

A recent survey in China showed a marked increase in the prevalence of T2DM, with a prevalence of $9.7 \%$, representing estimated 92.4 million adults in China with diabetes in 2007 .
In 2007, the prevalence of T2DM in China was almost $10 \%$, which was a three-fold increase in three decades [5]. Diabetes incidence, prevalence, and disease progression vary by ethnic group, as do dietary patterns; consequently, T2DM in the Chinese population is different from that in European and North American populations.

The Beijing Communities Diabetes Study (BCDS) is a clinical research project designed to investigate whether multifactorial interventions have a long-term effect on the risk of diabetesrelated macro-vascular complications in patients with T2DM living in urban communities of Beijing. It is the first of the kind ever undertaken in China. Using this large sample of patients with T2DM in the Beijing community, our aim is to identify and investigate the predictors of CVD in Asian persons with diabetics, with a focus on the Chinese poulation, in order to provide effective guidance for preventing CVD incidence. China is a developing country, and as such its medical resources are relatively inadequate. It is therefore important to determine how to compensate for the lack of medical resources in the long-term management of T2DM in terms of CVD prevention at the community level. The major feature of our research is the joint management model of community hospitals and top tier hospitals managing a large sample of patients with diabetes during a 10 -year follow-up. Our aim is to investigate whether achieving target control in patients with type 2 diabetes (blood glucose and blood pressure) can prevent CVD occurrence.

Persons with diabetes represent a special population in terms of asymptomatic cardiovascular risk assessment. Fortunately, the developmental process of CVD is slow and the incubation period is relatively long; as such, the study of cardiovascular risk assessment is of great significance for the prevention and treatment of CVD. The typical model of cardiovascular disease risk prediction was established by the Framingham Heart Study, and researchers subsequently developed a QRISK score based on the Framingham formula [6], which was then validated to be more consistent with the British population. A large prospective study published in 2009 found that the QRISK score 
underestimated 10-year CVD risk in subjects [7], while the Framingham risk assessment model overestimated the 10-year CVD risk in subjects. At the same time, some studies suggested that the model is not applicable in non-European countries. In order to establish a more suitable cardiovascular risk assessment model for Chinese patients, a 10-year ischemic cardiovascular disease risk assessment model and an annual risk assessment table with a simple assessment tool have been established, which have been shown to have satisfactory predictive value; these are recommended by domestic guidelines.

However, as yet there has been no research on a CVD risk assessment tool for patients with diabetes mellitus, with currently used tools often imitating or being borrowed from the above-mentioned risk assessment models. Because these earlier CVD risk assessment models were developed in the non-diabetic population and did not cover blood glucose control factors, whether the classical CVD risk assessment tool can truly reflect the CVD risk of patients with diabetes is not conclusive. In this context, it is necessary to explore CVD risk assessment tools for Chinese patients with diabetes. In the present study, we investigate the improved Framingham Risk Score (I-FRS) as a new cardiovascular risk assessment in persons with T2DM in the Beijing community and evaluate its effect on stratifying a patient's CVD risk when the efficacy of that patient's diabetes management is assessed.

\section{METHODS}

\section{Study Population and Intervention Methods}

Details of the BCDS study have been published previously [8]. For the present study (BCDS 12), all of the patients were attending 25 community health centers and were recruited between August 2008 and July 2009. The age of the patients ranged from 20 to 80 years, and all had been diagnosed with T2DM. The likelihood of the patients moving away from that their current Beijing community was taken into account when enrolling patients. The study was conducted in accordance with the Helsinki Declaration of 1964, as revised in 2013 and was approved by the Medical Ethics Committee of Beijing Tongren Hospital. Informed consent was obtained from all patients for being included in the study.

The patients enrolled in BCDS were monitored by 180 general practitioners (GPs) on a one-to-one basis during the 10-year multifactorial intervention [9]. The GPs were systemically educated weekly by six professors of endocrinology from top tier hospitals, thus enhancing the level of patient care by increasing regular clinical consultations.

The treatment goals in the BCDS are in accordance with 2007 and 2010 China guideline for type 2 diabetes, namely, a stepwise implementation of diet control, behavior modification, and optimum management strategy that targets hyperglycemia, hypertension, dyslipidemia, and microalbuminuria. A major focus is on comprehensive cardiovascular risk reduction, including monitoring of blood glucose, blood pressure, and lipid profiles. Patients who failed to achieve target goals were required to carry out intensive changes in lifestyle under the direction of the GPs; medication could be increased and/or a drug from a different class could be added to treatment. When difficult and/or complicated cases were encountered or the patients were in poor compliance with the current treatment regime, in order to optimize for treatment during the multifactorial intervention, the GPs repeatedly requested consultations. Where possible, all treatment decisions were to involve the patient, with a focus on patient preferences, needs, and values.

Approaches to the prevention of diabetic complications in the BCDS include the following: patients were seen at clinic visits every 2 months; glycated hemoglobin (HbA1c) levels were measured every 3-6 months; microalbumin urine test was performed annually [8]; dilated eye examinations were performed annually; the lipid profile determinations and electrocardiograms (ECGs) were performed at 6or 12-month intervals.

T2DM was defined according to the criteria of the World Health Organization. The study 
population consisted of patients with or without CVD. CVD was diagnosed in a top tier hospital and included coronary heart disease, myocardial infarction, angina pectoris, cerebrovascular disease, or other clinical manifestations of CVD. Cerebrovascular disease included ischemic attacks or strokes. Patients were considered to be hypertensive if they reported current use of antihypertensive medications and/or had a systolic blood pressure (SBP) $\geq 140 \mathrm{mmHg}$ and/or diastolic blood pressure $(\mathrm{DBP}) \geq 90 \mathrm{mmHg}$.

A total of 3232 adults with diabetes were enrolled in the study. The participants were categorized into a hypertension (HTN) group ( $n$ $=1436)$, a cardiovascular disease (CVD) group ( $n$ =929), and a no-complications diabetes mellitus (DM) group that had normal SBP/DBP and no CVD $(n=867)$. Patients with diabetes mellitus were followed up regularly to monitor the relevant indexes and calculate the I-FRS score, and actual CVD events were recorded.

All patients enrolled in this study had T2DM; however, the traditional FRS does not include blood glucose as a parameter. Therefore, based on the FRS [6], we applied the I-FRS to all patients at baseline to predict CVD risk using the following HbA1c risk categories: score $0=\mathrm{HbA} 1 \mathrm{c} \leq 7.0 \% ; \quad$ score $1=7.0 \%<\mathrm{HbA} 1 \mathrm{c}$ $\leq 7.9 \%$; score $2=8.0 \%<\mathrm{HbA} 1 \mathrm{c} \leq 8.9 \%$; and score $3=\mathrm{HbA} 1 \mathrm{c}>9.0 \%$. The receiving operating characteristic (ROC) curves of the I-FRS and the actual incidence of cardiovascular events were compared in the Beijing community diabetic population participation in the study. Subsequently, the effects of the interventions applied in reducing the cardiovascular risk scores were evaluated by comparing the 10-year follow-up I-FRS scores with the baseline scores.

On the basis of the baseline I-FRS, patients were stratified into cardiovascular risk strata of $<10 \%$ (low Framingham risk stratum), 10-20\% (medium Framingham risk stratum), and $>20 \%$ (high Framingham risk stratum) to evaluate the 10-year risk for CVD. For the present study, cardiovascular events were defined as the first nonfatal or fatal cardiovascular event, including myocardial infarction, sudden cardiac death, and nonfatal stroke.
Patients excluded from analysis in the present study included those with type 1 diabetes, urinary infections, hematuria, severe disabilities, hepatic failure, renal failure, schizophrenia, goiter or fever, sleep apnea syndrome.

\section{Study Procedures}

Baseline information on sociodemographic variables included age, gender, smoking habits, history of diabetes, and parental history of diabetes. Data obtained from clinical evaluations included height, body weight, body mass index (BMI), waist circumference, and hip circumference. Based on the Chinese guideline for the prevention and treatment of T2DM (2010 edition), overweight was defined as a BMI $>24 \mathrm{~kg} /$ $\mathrm{m}^{2}$. The neck circumference [10] at the upper margin of the laryngeal prominence was also measured with the patient's head erect and eyes facing forward. SBP and DBP values in all participants were also obtained.

\section{Laboratory Data}

Blood samples in all patients were drawn after an overnight fast. Laboratory measurements (using established methods) included:

1. Fasting plasma glucose (FPG), 2-h postprandial blood glucose, and fasting serum insulin concentrations. HbA1c (\%) was measured by a central endocrinology laboratory in Beijing Tongren Hospital using a Bio-Rad Variant hemoglobin analyzer (BioRad Laboratories, Inc., Hercules, CA, USA). Insulin resistance (determined by the homeostatic model assessment method) was calculated as the product of fasting insulin multiplied by fasting glucose divided by 22.5 .

2. Serum lipid concentrations (total cholesterol, low- and high-density lipoprotein [LDL and HDL]-cholesterols, and triglycerides.

3. Alanine aminotransferase, blood urea nitrogen, and serum creatinine concentrations. 
4. Urinary albumin excretion rate, which was performed via a centralized 8-h overnight urine collection.

After blood sampling, all patients underwent a resting 12-lead ECG. Angina pectoris and/ or $>0.1 \mathrm{mV}$ ST segment deviation during a maximal exercise stress test on a treadmill were recorded. Nonstereoscopic photographs of the central fundus were taken for all eyes (CR-DGi camera; Canon Inc, Tokyo, Japan).

On 31 December 2018, the participants were informed of the final results and asked to continue with normal medical care every year.

\section{Statistical Analyses}

The BCDS database was set up using a network database. Statistical analysis was performed using SAS ${ }^{\circledR}$ software (version 9.2; SAS Institute Inc., Cary, NC, USA). The results were expressed as means ( \pm standard deviation) or as medians with the range. Analysis of variance and the Chi-square test were used to compare continuous and categorical variables, respectively. The paired $t$ test was used to compare the I-FRS score at baseline and at the end of the follow-up. The ROC curve was used to test the prediction accuracy of the I-FRS on the actual incidence of CVD events. We used the Cox proportional hazards analysis to estimate hazards ratios of metabolic factors, with the 95\% confidence interval, for the effects of I-FRS on CVD risk. We included clinically important factors, such as age and sex, and significant factors, such as BP, $\mathrm{NC}$, and dyslipidemia. Kaplan-Meier analysis was used to assess the cumulative percentage of CVD events among different I-FRS groups by follow-up time, and then log-rank test was used to assess the difference among the I-FRS groups. All tests were two-sided, and the level of significance was established as $P<0.05$.

\section{RESULTS}

Among the 3232 patients with T2DM who were included in the analysis, 204 (mean age $45.2 \pm 8.8$ years) were categorized in the low FRS stratum (I-FRS $<10 \%), 2531$ (mean age
$63.5 \pm 9.1$ years ) were categorized in the medium FRS stratum (I-FRS 10-20\%), and 497 (mean age $72.0 \pm 6.3$ years) were categorized in the high FRS stratum (I-FRS $>20 \%$ ). The clinical characteristics, such as the frequency of smoking and use of metformin ratios, were all significantly different among the three groups (Table 1).

\section{Patients' Clinical Characteristics}

The baseline and follow-up demographic and clinical characteristics of patients in the low, medium and high FRS strata are shown in Table 1 . The patients in medium and high FRS strata had the highest mean age. At baseline and at the post-intervention evaluation, patients in the medium and high FRS strata had higher SBP values than those in the low FRS stratum $(P$ $<0.01)$. Patients in the high FRS stratum showed a tendency towards a markedly smaller neck circumference than did those in the low and medium FRS strata, both at baseline $(35.7 \pm 3.4$ vs. $36.8 \pm 3.7$ and $36.6 \pm 3.8 \mathrm{~cm}$, respectively; $P<0.05$ ) and after the 10 -year follow-up $(35.5 \pm 3.3$ vs. $36.7 \pm 3.4$ and $36.6 \pm 3.5 \mathrm{~cm}$, respectively; $P<0.05$ ) (Table 2).

For glucose metabolism parameters and a number of the hemodynamic values (lipid and serum creatinine concentrations), the patients in the medium and high FRS strata were more likely to have higher FPG and HbA1c levels and a more adverse lipid profile than those in the low FRS stratum (all $P<0.01$ ). After the 10-year follow-up, there were no significant differences in these variables among the three groups (Table 2). There were no significant differences in uric acid levels at baseline and at the end of the follow-up.

\section{Changes in Patients' FRS}

Patients in the medium and high FRS strata had significantly higher I-FRS than those in the low FRS stratum, both at baseline and after the 10 -year follow-up (both $P<0.01$ ). The post-intervention I-FRS in all three groups were significantly lower compared with the respective baseline scores $(P<0.01$ for each) (Fig. 1). 
Table 1 Demographic and clinical characteristics of patients in the low, medium, and high Framingham Risk Score strata at baseline (2008) in the Beijing Communities Diabetes Study (Report 22)

\begin{tabular}{|c|c|c|c|c|c|c|}
\hline \multirow{2}{*}{$\begin{array}{l}\text { Patients } \\
\text { characteristics }\end{array}$} & \multicolumn{4}{|c|}{ Framingham Risk Score stratification ${ }^{a}$} & \multirow{2}{*}{$\begin{array}{l}\text { Statistical } \\
\text { quantity }^{\mathbf{b}}\end{array}$} & \multirow[t]{2}{*}{$P$ value $^{\mathrm{c}}$} \\
\hline & $\begin{array}{l}\text { Total } \\
(n=3232 \\
\text { patients })\end{array}$ & $\begin{array}{l}\text { I-FRS }<10 \% \\
(n=204 \\
\text { patients })\end{array}$ & $\begin{array}{l}\text { I-FRS 10-20\% } \\
(n=2531 \\
\text { patients })\end{array}$ & $\begin{array}{l}\text { I-FRS }>20 \% \\
(n=497 \\
\text { patients })\end{array}$ & & \\
\hline Age (years) & $63.7 \pm 10.4$ & $45.2 \pm 8.8$ & $63.5 \pm 9.1^{*}$ & $72.0 \pm 6.3^{* \dagger}$ & $694.40(\mathrm{~F})$ & $<0.001$ \\
\hline \multicolumn{7}{|l|}{ Gender } \\
\hline Male & $1295(40.07 \%)$ & $114(55.88 \%)$ & $1170(46.23 \%)$ & $11(2.21 \%)$ & $\begin{array}{c}357.80 \\
\left(\chi^{2}\right)\end{array}$ & $<0.001$ \\
\hline Female & $1937(59.93 \%)$ & $90(44.12 \%)$ & $1361(53.77 \%)$ & $486(97.79 \%)$ & & \\
\hline $\begin{array}{l}\text { Duration of diabetes } \\
\text { (years) }\end{array}$ & $3.8[0.0,9.8]$ & $1.8[0.0,5.2]$ & $3.8[0.0,9.7]^{*}$ & $5.0[0.0,11.8]^{* \dagger}$ & $6.51(\mathrm{Z})$ & $<0.001$ \\
\hline BMI $\left(\mathrm{kg} / \mathrm{m}^{2}\right)$ & $25.4 \pm 3.5$ & $25.8 \pm 4.0$ & $25.3 \pm 3.5$ & $25.3 \pm 3.4$ & $1.86(\mathrm{~F})$ & 0.156 \\
\hline $\begin{array}{l}\text { Waist circumference } \\
(\mathrm{cm})\end{array}$ & $89.1 \pm 9.4$ & $88.6 \pm 10.0$ & $89.1 \pm 9.3$ & $89.3 \pm 9.4$ & $0.38(\mathrm{~F})$ & 0.684 \\
\hline $\begin{array}{l}\text { Hip } \\
\quad \text { circumference }(\mathrm{cm})\end{array}$ & $99.5 \pm 8.6$ & $99.9 \pm 8.7$ & $99.4 \pm 8.5$ & $99.9 \pm 8.6$ & $0.96(\mathrm{~F})$ & 0.383 \\
\hline Waist-hip ratio & $0.90 \pm 0.06$ & $0.89 \pm 0.06$ & $0.90 \pm 0.06$ & $0.89 \pm 0.07$ & $2.77(\mathrm{~F})$ & 0.063 \\
\hline $\begin{array}{l}\text { Neck circumference } \\
\quad(\mathrm{cm})\end{array}$ & $36.5 \pm 3.8$ & $36.8 \pm 3.7$ & $36.6 \pm 3.8$ & $35.7 \pm 3.4^{* \dagger}$ & $11.68(\mathrm{~F})$ & $<0.001$ \\
\hline SBP $(\mathrm{mmHg})$ & $129.5 \pm 14.7$ & $121.0 \pm 12.1$ & $128.2 \pm 14.1^{*}$ & $139.7 \pm 13.8^{* \dagger}$ & $182.55(\mathrm{~F})$ & $<0.001$ \\
\hline $\mathrm{DBP}(\mathrm{mmHg})$ & $78.1 \pm 8.8$ & $78.6 \pm 8.1$ & $78.0 \pm 8.8$ & $78.0 \pm 9.0$ & $0.50(\mathrm{~F})$ & 0.608 \\
\hline \multicolumn{7}{|l|}{ Smoker } \\
\hline No & $2718(84.10 \%)$ & $187(91.67 \%)$ & $2092(82.66 \%)$ & $439(88.33 \%)$ & $19.33\left(\chi^{2}\right)$ & $<0.001$ \\
\hline Yes & $514(15.90 \%)$ & $17(8.33 \%)$ & $439(17.34 \%)$ & $58(11.67 \%)$ & & \\
\hline $\mathrm{FPG}(\mathrm{mmol} / \mathrm{L})$ & $7.86 \pm 2.63$ & $7.57 \pm 2.63$ & $7.81 \pm 2.61$ & $8.27 \pm 2.68^{* \dagger}$ & $7.86(\mathrm{~F})$ & $<0.001$ \\
\hline $\mathrm{Hpg}(\mathrm{mmol} / \mathrm{L})$ & $10.71 \pm 4.99$ & $10.25 \pm 3.84$ & $10.65 \pm 5.15$ & $11.24 \pm 4.58^{* \dagger}$ & $3.75(\mathrm{~F})$ & 0.024 \\
\hline HbAlc (\%) & $7.3 \pm 1.6$ & $7.2 \pm 1.8$ & $7.3 \pm 1.6$ & $7.8 \pm 1.6^{* \dagger}$ & $22.30(\mathrm{~F})$ & $<0.001$ \\
\hline $\mathrm{TG}(\mathrm{mmol} / \mathrm{L})$ & $1.91 \pm 1.40$ & $1.86 \pm 1.38$ & $1.88 \pm 1.37$ & $2.11 \pm 1.56^{* \dagger}$ & $5.62(\mathrm{~F})$ & 0.004 \\
\hline $\mathrm{TC}(\mathrm{mmol} / \mathrm{L})$ & $5.20 \pm 1.23$ & $4.50 \pm 0.92$ & $5.16 \pm 1.21^{*}$ & $5.72 \pm 1.24^{*^{\dagger}}$ & $82.67(\mathrm{~F})$ & $<0.001$ \\
\hline $\begin{array}{l}\text { HDL-cholesterol } \\
\qquad(\mathrm{mmol} / \mathrm{L})\end{array}$ & $1.34 \pm 0.43$ & $1.22 \pm 0.32$ & $1.33 \pm 0.42^{*}$ & $1.42 \pm 0.48^{* \dagger}$ & $16.98(\mathrm{~F})$ & $<0.001$ \\
\hline $\begin{array}{l}\text { LDL-cholesterol } \\
\qquad(\mathrm{mmol} / \mathrm{L})\end{array}$ & $2.95 \pm 0.86$ & $2.54 \pm 0.77$ & $2.92 \pm 0.83^{*}$ & $3.24 \pm 0.95^{* \dagger}$ & $54.88(\mathrm{~F})$ & $<0.001$ \\
\hline $\begin{array}{l}\text { Alanine } \\
\text { aminotransferase } \\
(\mathrm{U} / \mathrm{L})\end{array}$ & $22.54 \pm 14.69$ & $25.04 \pm 16.66$ & $22.92 \pm 14.77^{*}$ & $19.49 \pm 12.85^{* \dagger}$ & $12.86(\mathrm{~F})$ & $<0.001$ \\
\hline
\end{tabular}


Table 1 continued

\begin{tabular}{|c|c|c|c|c|c|c|}
\hline \multirow{2}{*}{$\begin{array}{l}\text { Patients } \\
\text { characteristics }\end{array}$} & \multicolumn{4}{|c|}{ Framingham Risk Score stratification $^{a}$} & \multirow{2}{*}{$\begin{array}{l}\text { Statistical } \\
\text { quantity }^{b}\end{array}$} & \multirow[t]{2}{*}{$P$ value $^{\mathrm{c}}$} \\
\hline & $\begin{array}{l}\text { Total } \\
(n=3232 \\
\text { patients })\end{array}$ & $\begin{array}{l}\text { I-FRS }<10 \% \\
(n=204 \\
\text { patients })\end{array}$ & $\begin{array}{l}\text { I-FRS 10-20\% } \\
(n=2531 \\
\text { patients })\end{array}$ & $\begin{array}{l}\text { I-FRS }>20 \% \\
(n=497 \\
\text { patients })\end{array}$ & & \\
\hline $\begin{array}{l}\text { Blood urea nitrogen } \\
(\mathrm{mmol} / \mathrm{L})\end{array}$ & $5.3[4.3,6.5]$ & $4.9[3.9,6.1]$ & $5.3[4.3,6.5]^{*}$ & $5.8[4.6,6.9]^{*^{\dagger}}$ & $5.13(Z)$ & $<0.001$ \\
\hline $\begin{array}{l}\text { Creatinine (mmol/ } \\
\text { L) }\end{array}$ & $\begin{array}{c}74.0[59.7, \\
88.4]\end{array}$ & $75.0[61.0,89.2]$ & $74.1[60.0,88.6]$ & $70.5[57.6,85.6]$ & $2.83(\mathrm{Z})$ & 0.018 \\
\hline $\begin{array}{l}\text { Urine acid (mmol/ } \\
\text { L) }\end{array}$ & $\begin{array}{l}279.6[222.0, \\
\quad 344.6]\end{array}$ & $\begin{array}{l}260.0[207.9, \\
330.0]\end{array}$ & $\begin{array}{l}279.8[222.7 \\
346.0]\end{array}$ & $\begin{array}{l}282.1[226.5 \\
337.4]\end{array}$ & $2.05(Z)$ & 0.122 \\
\hline $\begin{array}{l}\text { Improved } \\
\text { Framingham risk } \\
\text { score (I-FRS) }\end{array}$ & $15.56 \pm 4.01$ & $6.37 \pm 3.28$ & $15.15 \pm 2.36^{*}$ & $21.40 \pm 1.45^{* \dagger}$ & $\begin{array}{c}3217.11 \\
(F)\end{array}$ & $<0.001$ \\
\hline \multicolumn{7}{|l|}{ History of disease ${ }^{\mathrm{d}}$} \\
\hline $\mathrm{DM}$ & $867(26.83 \%)$ & $124(60.78 \%)$ & $723(28.57 \%)$ & $20(4.02 \%)$ & $\begin{array}{c}262.29 \\
\left(\chi^{2}\right)\end{array}$ & $<0.001$ \\
\hline HTN & $1436(44.43 \%)$ & $62(30.39 \%)$ & $1097(43.34 \%)$ & $277(55.73 \%)$ & & \\
\hline CVD & $929(28.74 \%)$ & $18(8.82 \%)$ & $711(28.09 \%)$ & $200(40.24 \%)$ & & \\
\hline $\begin{array}{l}\text { Antihypertensive } \\
\text { therapy }\end{array}$ & $1732(53.59 \%)$ & $47(23.04 \%)$ & $1270(50.18 \%)$ & $415(83.50 \%)$ & $\begin{array}{c}267.19 \\
\left(\chi^{2}\right)\end{array}$ & $<0.001$ \\
\hline $\begin{array}{l}\text { Hypoglycemic } \\
\text { therapy }\end{array}$ & $2928(90.59 \%)$ & $169(82.84 \%)$ & $2304(91.03 \%)$ & $455(91.55 \%)$ & $15.48\left(\chi^{2}\right)$ & $<0.001$ \\
\hline Sulfonylurea drugs & $1962(60.71 \%)$ & $115(56.37 \%)$ & $1555(61.44 \%)$ & $292(58.75 \%)$ & $2.97\left(\chi^{2}\right)$ & 0.227 \\
\hline Metformin & $1430(44.25 \%)$ & $73(35.78 \%)$ & $1127(44.53 \%)$ & $230(46.28 \%)$ & $6.83\left(\chi^{2}\right)$ & 0.033 \\
\hline Insulin therapy & 2097 (64.88\%) & $120(58.82 \%)$ & $1664(65.74 \%)$ & $313(62.98 \%)$ & $4.90\left(\chi^{2}\right)$ & 0.086 \\
\hline
\end{tabular}

Values are expressed as the mean \pm standard deviation $(\mathrm{SD})$; the median with the first and third quartiles (Q1,Q3) in square brackets; or a number with the percentage in parenthesis

$B M I$ Body mass index, $D B P$ diastolic blood pressure, $F P G$ fasting plasma glucose, $H b A 1 c$ hemoglobin Alc, $H D L-C$ highdensity lipoprotein-cholesterol, $H p g$ 2-h postprandial blood glucose, $I-F R S$ improved Framingham Risk Score, $L D L-C$ lowdensity lipoprotein-cholesterol $S B P$ systolic blood pressure, $T C$ total cholesterol, $T G$ triglyceride

a On the basis of the baseline I-FRS, patients were stratified into cardiovascular risk categories of $<10 \%$ (low FRS stratum), $10-20 \%$ (medium FRS stratum), and $>20 \%$ (high FRS stratum)

b F, Fisher's exact test; $\chi^{2}$, Chi-square test; $Z, Z$ test

c Statistical significance of the differences among the three groups. Asterisk $\left(^{*}\right)$ is vs. low FRS stratum; dagger $\left(^{\dagger}\right)$ is vs. medium FRS stratum

d In terms of disease, participants were categorized into a hypertension (HTN) group, a cardiovascular disease (CVD) group, and a no-complications diabetes mellitus (DM) group with normal SBP/DBP and no CVD $(n=867)$. 
Table 2 Demographic and clinical characteristics of patients in the low, medium, and high Framingham Risk Score strata at the end of the study follow-up (2018) in the Beijing Communities Diabetes Study (Report 22)

\begin{tabular}{|c|c|c|c|c|c|}
\hline \multirow{2}{*}{$\begin{array}{l}\text { Patient } \\
\text { characteristics }\end{array}$} & \multicolumn{3}{|c|}{ Framingham Risk Score stratification ${ }^{a}$} & \multirow{2}{*}{$\begin{array}{l}\text { Statistical } \\
\text { quantity }\end{array}$} & \multirow[t]{2}{*}{$P$ value } \\
\hline & $\begin{array}{l}\text { I-FRS }<10 \% \\
(n=204 \text { patients })\end{array}$ & $\begin{array}{l}\text { I-FRS 10-20\% } \\
(n=2531 \text { patients })\end{array}$ & $\begin{array}{l}\text { I-FRS }>20 \%(n \\
=497 \text { patients })\end{array}$ & & \\
\hline BMI $\left(\mathrm{kg} / \mathrm{m}^{2}\right)$ & $25.4 \pm 3.6$ & $25.2 \pm 3.3$ & $25.3 \pm 3.2$ & $0.36(\mathrm{~F})$ & 0.699 \\
\hline $\begin{array}{l}\text { Waist circumference } \\
(\mathrm{cm})\end{array}$ & $88.0 \pm 9.7$ & $87.7 \pm 8.5$ & $87.4 \pm 8.5$ & $0.21(\mathrm{~F})$ & 0.808 \\
\hline $\begin{array}{l}\text { Hip } \\
\quad \text { circumference }(\mathrm{cm})\end{array}$ & $98.8 \pm 9.9$ & $97.7 \pm 8.3$ & $97.2 \pm 8.0$ & $1.55(\mathrm{~F})$ & 0.212 \\
\hline $\begin{array}{l}\text { Neck circumference } \\
\quad(\mathrm{cm})\end{array}$ & $36.7 \pm 3.4$ & $36.6 \pm 3.5$ & $35.5 \pm 3.3^{*^{\dagger}}$ & $11.26(\mathrm{~F})$ & $<0.001$ \\
\hline $\mathrm{SBP}(\mathrm{mmHg})$ & $125.3 \pm 9.6$ & $125.6 \pm 8.3$ & $128.6 \pm 9.3^{* \dagger}$ & $14.46(\mathrm{~F})$ & $<0.001$ \\
\hline $\mathrm{DBP}(\mathrm{mmHg})$ & $74.6 \pm 6.6$ & $73.8 \pm 6.4 a$ & $72.5 \pm 7.4^{*^{\dagger}}$ & $5.86(\mathrm{~F})$ & 0.003 \\
\hline FPG $(\mathrm{mmol} / \mathrm{L})$ & $7.56 \pm 2.41$ & $7.59 \pm 2.11$ & $7.66 \pm 2.18$ & $0.13(\mathrm{~F})$ & 0.876 \\
\hline $\mathrm{Hpg}(\mathrm{mmol} / \mathrm{L})$ & $9.26 \pm 2.39$ & $9.17 \pm 2.03$ & $9.16 \pm 2.07$ & $0.08(\mathrm{~F})$ & 0.923 \\
\hline HbAlc (\%) & $7.07 \pm 1.37$ & $7.09 \pm 1.16$ & $7.14 \pm 1.12$ & $0.19(\mathrm{~F})$ & 0.831 \\
\hline $\mathrm{TG}(\mathrm{mmol} / \mathrm{L})$ & $1.60 \pm 0.85$ & $1.56 \pm 0.77$ & $1.75 \pm 0.84$ & $5.44(\mathrm{~F})$ & 0.004 \\
\hline $\mathrm{TC}(\mathrm{mmol} / \mathrm{l})$ & $4.62 \pm 1.00$ & $4.47 \pm 1.00$ & $4.75 \pm 1.10 \dagger$ & $8.09(\mathrm{~F})$ & $<0.001$ \\
\hline $\begin{array}{l}\text { HDL-cholesterol } \\
(\mathrm{mmol} / \mathrm{L})\end{array}$ & $1.35 \pm 0.36$ & $1.33 \pm 0.47$ & $1.35 \pm 0.32$ & $0.20(\mathrm{~F})$ & 0.819 \\
\hline $\begin{array}{l}\text { LDL-cholesterol } \\
\qquad(\mathrm{mmol} / \mathrm{L})\end{array}$ & $2.61 \pm 0.78$ & $2.45 \pm 0.73$ & $2.57 \pm 0.86$ & $4.20(\mathrm{~F})$ & 0.015 \\
\hline $\begin{array}{l}\text { Alanine } \\
\text { aminotransferase } \\
(\mathrm{U} / \mathrm{L})\end{array}$ & $19.2[15.0,25.0]$ & $19.0[15.0,24.0]$ & $19.0[14.3,23.0]$ & $0.59(\mathrm{Z})$ & 0.838 \\
\hline $\begin{array}{l}\text { Blood urea nitrogen } \\
(\mathrm{mmol} / \mathrm{L})\end{array}$ & $5.6[4.6,6.3]$ & $5.6[4.8,6.6]$ & $6.1[5.2,7.3]^{*^{\dagger}}$ & $5.23(\mathrm{Z})$ & $<0.001$ \\
\hline $\begin{array}{l}\text { Creatinine (mmol/ } \\
\text { L) }\end{array}$ & $68.0[57.0,77.6]$ & $71.0[60.0,83.2]$ & $71.0[60.0,83.1]$ & $1.75(\mathrm{Z})$ & 0.218 \\
\hline Urine acid $(\mathrm{mmol} / \mathrm{l})$ & $300.0[250.7,355.0]$ & $306.0[251.0,358.0]$ & $296.5[241.0,353.0]$ & $1.07(\mathrm{Z})$ & 0.563 \\
\hline $\begin{array}{l}\text { Hypoglycemic } \\
\text { therapy }\end{array}$ & $115(90.55 \%)$ & $1524(94.78 \%)$ & $279(95.55 \%)$ & $4.70\left(\chi^{2}\right)$ & 0.095 \\
\hline Sulfonylurea drugs & $18(14.17 \%)$ & $313(19.47 \%)$ & $52(17.81 \%)$ & $2.41\left(\chi^{2}\right)$ & 0.299 \\
\hline Metformin & $71(55.91 \%)$ & $737(45.83 \%)$ & $105(35.96 \%)$ & $16.19\left(\chi^{2}\right)$ & $<0.001$ \\
\hline
\end{tabular}


Table 2 continued

\begin{tabular}{|c|c|c|c|c|c|}
\hline \multirow{2}{*}{$\begin{array}{l}\text { Patient } \\
\text { characteristics }\end{array}$} & \multicolumn{3}{|c|}{ Framingham Risk Score stratification ${ }^{a}$} & \multirow{2}{*}{$\begin{array}{l}\text { Statistical } \\
\text { quantity }^{b}\end{array}$} & \multirow[t]{2}{*}{$P$ value } \\
\hline & $\begin{array}{l}\text { I-FRS }<10 \% \\
(n=204 \text { patients })\end{array}$ & $\begin{array}{l}\text { I-FRS } \sim 10-20 \% \\
(n=2531 \text { patients })\end{array}$ & $\begin{array}{l}\text { I-FRS }>20 \%(n \\
=497 \text { patients) }\end{array}$ & & \\
\hline Insulin therapy & $28(22.05 \%)$ & $443(27.55 \%)$ & $89(30.48 \%)$ & $3.17\left(\chi^{2}\right)$ & 0.205 \\
\hline
\end{tabular}

Values are expressed as the mean $\pm \mathrm{SD}$; the median with the first and third quartiles $(\mathrm{Q} 1, \mathrm{Q} 3)$ in square brackets; or a number with the percentage in parenthesis

a For explanation of I-FRS stratification, see footnote to Table 1

b F, Fisher's exact test; $\chi^{2}$, Chi-square test; $Z, Z$ test

c Statistical significance of the differences among the three groups. Asterisk $\left(^{*}\right)$ is vs. low FRS stratum; dagger $\left({ }^{\dagger}\right)$ is vs. medium FRS stratum

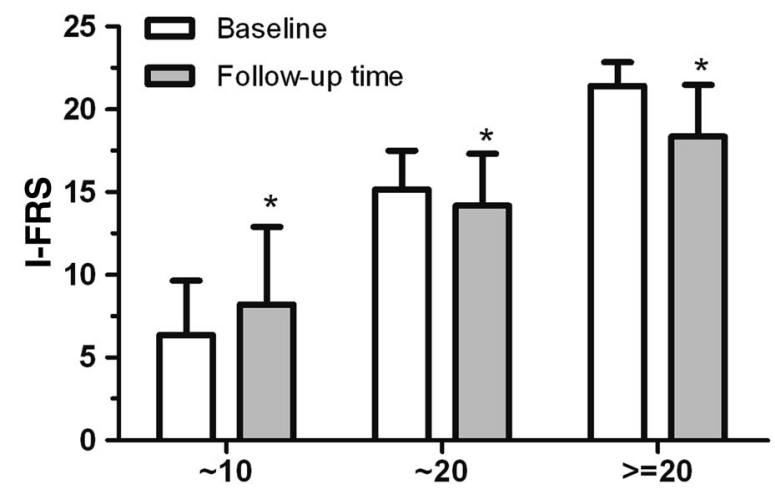

Fig. 1 Improved Framingham Risk Score (I-FRS). Changes in the I-FRS according to low $(\sim 10 \%)$, medium $(\sim 10-20 \%)$, and high $(>20 \%)$ FRS strata for cardiovascular disease from baseline to end of the 10-year followup. Asterisk indicates significant difference at $P<0.01$ versus baseline (2008)

Regarding the I-FRS in the DM, HTN and CVD groups, the I-FRS were significantly lower at the end of the 10-year follow-up in the HTN and CVD groups; this correlation was not detected in the DM group (Fig. 2).

\section{All-Cause Endpoint Events after 10-Years of Follow-Up}

The ROC curve of I-FRS $(P=0.000)$ showed a statistically significant prediction accuracy for the actual incidence of CVD events. During the 10 -year follow-up period, the cumulative cardiovascular event rate was $14.49 \%$ for the high FRS stratum and $10.51 \%$ for the medium FRS

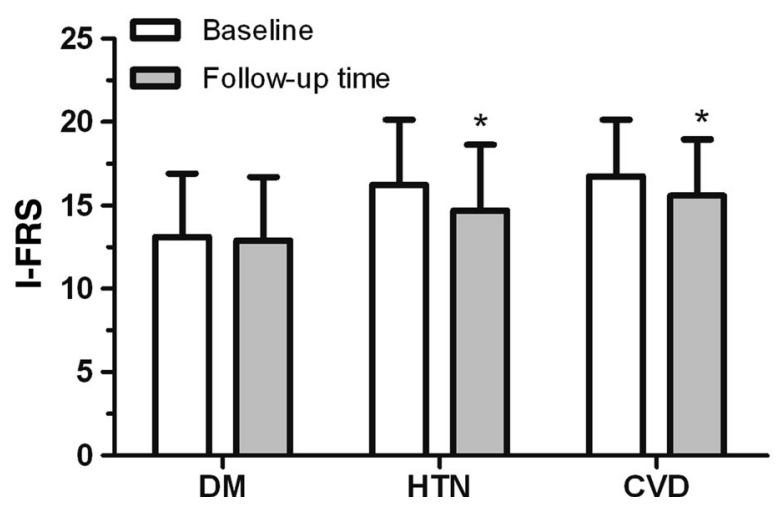

Fig. 2 Improved Framingham risk scores. Changes in the I-FRS according to disease categories from baseline to the end of the 10-year follow-up. Asterisk indicates significant difference at $P<0.01$ versus baseline (2008). HTN Hypertension group, $C V D$ cardiovascular disease group, $D M$ no-complications diabetes mellitus group with normal systolic/diastolic blood pressure

(Table 3); in comparison, the cumulative cardiovascular event rate was significantly lower in the low Framingham risk stratum $(2.46 \% ; P$ $<0.001$ ) (Fig. 3). No first cardiovascular events were diagnosed only by ECG, as all had previously been diagnosed clinically.

Factors analysis was performed to explore the variables contributing to the incidence of endpoint events. In the initial regression model, age, gender, SBP, FPG, and lipid profiles were considered to be potential confounding factors (Table 4). In the multivariate analyses that were controlled for age and clustering by clinic, patients in the medium and high FRS strata had 
Table 3 The incidence of endpoint events among the three cardiovascular risk strata distinguished by the improved Framingham Risk Score

\begin{tabular}{|c|c|c|c|c|c|c|}
\hline \multirow[t]{2}{*}{ Endpoint events } & \multirow{2}{*}{$\begin{array}{l}\text { Total } \\
(n=3232 \\
\text { patients })\end{array}$} & \multicolumn{3}{|c|}{ Framingham Risk Score stratification ${ }^{a}$} & \multirow{2}{*}{$\begin{array}{l}\text { Statistical } \\
\text { quantity }\end{array}$} & \multirow[t]{2}{*}{$P$ value } \\
\hline & & $\begin{array}{l}\text { I-FRS }<10 \% \\
(n=204 \\
\text { patients })[/ 100 \\
\text { person-year }]\end{array}$ & $\begin{array}{l}\text { I-FRS } \sim 10-20 \% \\
(n=2531 \\
\text { patients) }[/ 100 \\
\text { person-year] }\end{array}$ & $\begin{array}{l}\text { I-FRS }>20 \% \\
(n=497 \\
\text { patients })[/ 100 \\
\text { person-year }]\end{array}$ & & \\
\hline CVD events & $343(10.61)$ & $5(2.45)[0.25]$ & $266(10.51)[1.05]$ & $72(14.49)[1.45]$ & $22.22\left(\chi^{2}\right)$ & $<0.001$ \\
\hline Cardiovascular & $201(6.22)$ & $5(2.45)[0.25]$ & $158(6.24)[0.62]$ & $38(7.65)[0.76]$ & $6.70\left(\chi^{2}\right)$ & 0.035 \\
\hline $\begin{array}{l}\text { Myocardial } \\
\text { infarction }\end{array}$ & $58(1.79)$ & $1(0.49)[0.05]$ & $49(1.94)[0.19]$ & $8(1.61)[0.16]$ & $2.35\left(\chi^{2}\right)$ & 0.308 \\
\hline $\begin{array}{l}\text { Unstable angina } \\
\text { pectoris }\end{array}$ & $28(0.87)$ & $3(1.47)[0.15]$ & $22(0.87)[0.09]$ & $3(0.60)[0.06]$ & $0.03(\mathrm{~F})$ & 0.451 \\
\hline $\begin{array}{l}\text { Install cardiac } \\
\text { pacemaker }\end{array}$ & $6(0.19)$ & $0(0.00)[0.00]$ & $6(0.24)[0.02]$ & $0(0.00)[0.00]$ & $0.23(\mathrm{~F})$ & 0.727 \\
\hline $\begin{array}{l}\text { Coronary stent } \\
\text { implantation }\end{array}$ & $50(1.55)$ & $1(0.49)[0.05]$ & $40(1.58)[0.16]$ & $9(1.81)[0.18]$ & $1.74\left(\chi^{2}\right)$ & 0.419 \\
\hline $\begin{array}{l}\text { Coronary artery } \\
\text { bypass grafting }\end{array}$ & $17(0.53)$ & $0(0.00)[0.00]$ & $14(0.55)[0.06]$ & $3(0.60)[0.06]$ & $0.08(\mathrm{~F})$ & 0.732 \\
\hline $\begin{array}{l}\text { Heart failure } \\
\text { hospitalization }\end{array}$ & $21(0.65)$ & $0(0.00)[0.00]$ & $12(0.47)[0.05]$ & $9(1.81)[0.18]$ & $0.00(\mathrm{~F})$ & 0.006 \\
\hline Cerebrovascular & $142(4.39)$ & $0(0.00)[0.00]$ & $108(4.27)[0.43]$ & $34(6.84)[0.68]$ & $16.56\left(\chi^{2}\right)$ & $<0.001$ \\
\hline $\begin{array}{l}\text { Acute cerebral } \\
\text { hemorrhage }\end{array}$ & $18(0.56)$ & $0(0.00)[0.00]$ & $16(0.63)[0.06]$ & $2(0.40)[0.04]$ & $0.07(\mathrm{~F})$ & 0.743 \\
\hline Lacunar infarction & $30(0.93)$ & $0(0.00)[0.00]$ & $25(0.99)[0.10]$ & $5(1.01)[0.10]$ & $0.03(\mathrm{~F})$ & 0.483 \\
\hline $\begin{array}{l}\text { Acute cerebral } \\
\text { infarction with } \\
\text { limb movement } \\
\text { disorder }\end{array}$ & $69(2.13)$ & $0(0.00)[0.00]$ & $46(1.82)[0.18]$ & $23(4.63)[0.46]$ & $20.45\left(\chi^{2}\right)$ & $<0.001$ \\
\hline $\begin{array}{l}\text { Transient ischemic } \\
\text { attack (TIA) }\end{array}$ & $22(0.68)$ & $0(0.00)[0.00]$ & $19(0.75)[0.08]$ & $3(0.60)[0.06]$ & $0.05(\mathrm{~F})$ & 0.717 \\
\hline $\begin{array}{l}\text { Cardiac and cerebral } \\
\text { vascular death }\end{array}$ & $90(2.78)$ & $0(0.00)[0.00]$ & $67(2.65)[0.26]$ & $23(4.63)[0.46]$ & $12.26\left(\chi^{2}\right)$ & 0.002 \\
\hline Cardiac death & $63(1.95)$ & $0(0.00)[0.00]$ & $45(1.78)[0.18]$ & $18(3.62)[0.36]$ & $11.72\left(\chi^{2}\right)$ & 0.003 \\
\hline Cerebral death & $27(0.84)$ & $0(0.00)[0.00]$ & $22(0.87)[0.09]$ & $5(1.01)[0.10]$ & $0.03(\mathrm{~F})$ & 0.447 \\
\hline
\end{tabular}

Values in table are presented as the number of patients with the percentage in parenthesis and the $/ 100$ person-year statistic given in square brackets

${ }^{a}$ For explanation of I-FRS stratification, see footnote to Table 1

${ }^{b}$ F, Fisher's exact test; $\chi^{2}$, Chi-square test 

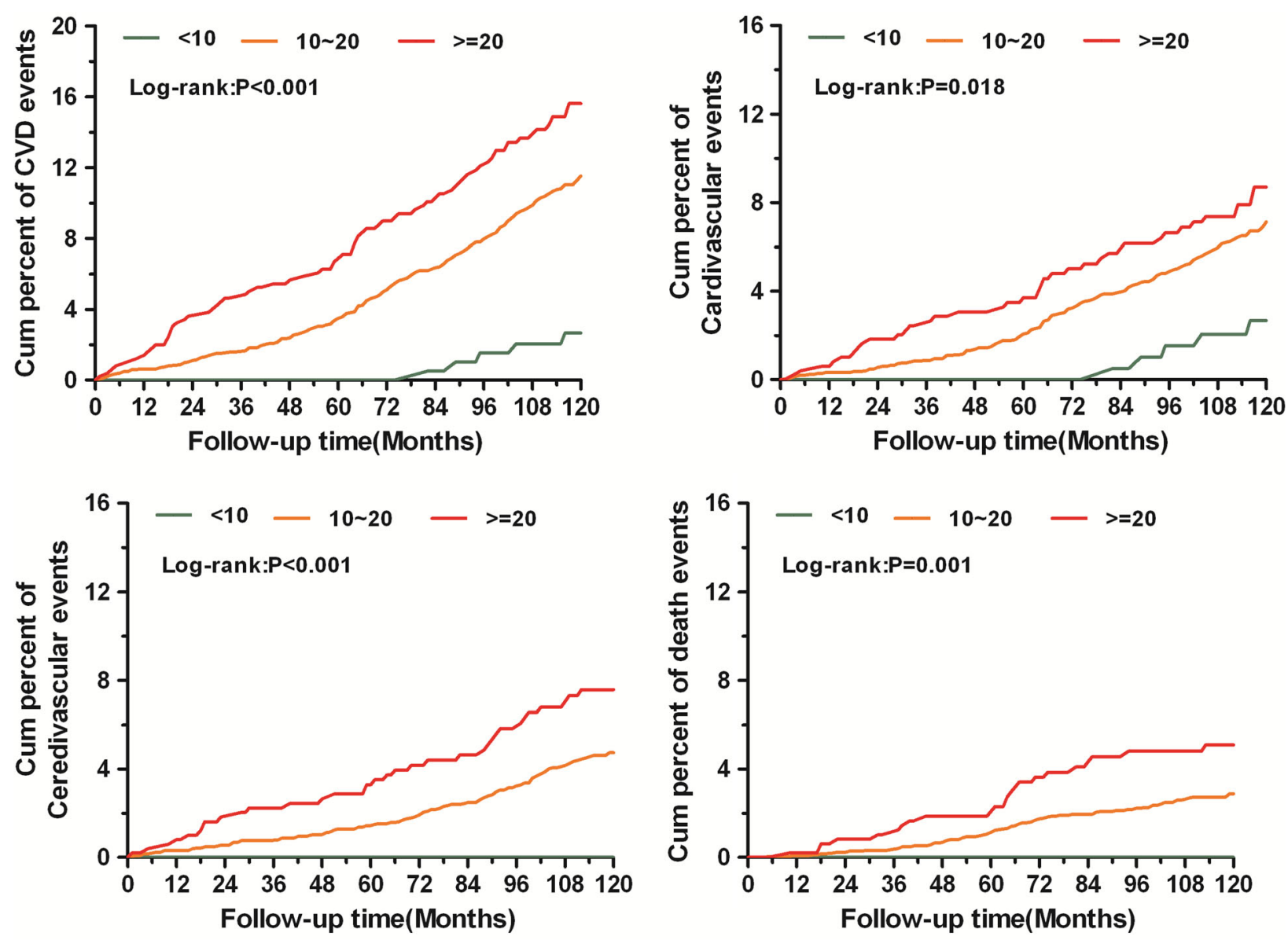

Fig. 3 Cumulative incidences of all-cause endpoint events during the follow-up in the low, medium, and high FRS strata. Cum cumulative, CVD cardiovascular disease

a higher incidence of endpoint events than those in the low FRS stratum $(P<0.05-0.01$, respectively) (Table 5). In a analysis of the effects of the I-FRS on the incidence of CVD events, the 10-year hazard ratio (HR) for CVD events in diabetic patients with I-FRS of $>20 \%$ was 12.5-fold higher than that for patients with an I-FRS of $<10 \%$. When calculated by I-FRS score, the 10-year HRs for death caused by CVD events in diabetic patients with I-FRS of $>20 \%$ was 2.91-fold higher than that of patients with I-FRS of $<10 \%$ (Fig. 3 ). A decrease in the BMI was found to be closely related to endpoint events, including all-cause death (especially cardiac and cerebral vascular death), myocardial infarction, coronary stent implantation, and coronary artery bypass grafting (Table 6).

\section{DISCUSSION}

This longitudinal study lasted 10 years and was based on community health center-hospital integrated management of T2DM. All treatments administered in the BCDS were in accordance with national guidelines for the management of T2DM in China. Furthermore, the inclusion of regular clinical consultations by professors in endocrinology from top tier hospitals in disease management is unique to this study. The co-operation between the systematically trained GPs and the professors in endocrinology has formed the basis for the efficient implementation of this multifactorial intervention during the last 10 years. It represents a unique healthcare model with Chinese characteristics. The joint management model of 
Table 4 Effects of different clinical characteristics on the hazard ratio of endpoint events

\begin{tabular}{|c|c|c|c|c|}
\hline Variables & CVD events & $\begin{array}{l}\text { Cardiovascular } \\
\text { events }\end{array}$ & $\begin{array}{l}\text { Cerebrovascular } \\
\text { events }\end{array}$ & $\begin{array}{l}\text { Cardiac and cerebral } \\
\text { vascular death }\end{array}$ \\
\hline Gender & $0.73(0.59-0.90)$ & $0.70(0.53-0.92)$ & $0.77(0.56-1.08)$ & $0.44(0.29-0.67)$ \\
\hline Age (years) & $1.03(1.02-1.05)$ & $1.04(1.02-1.05)$ & $1.03(1.01-1.05)$ & $1.10(1.07-1.13)$ \\
\hline Duration of diabetes (years) & $1.02(1.00-1.03)$ & $1.02(1.00-1.04)$ & $1.02(0.99-1.04)$ & $1.04(1.01-1.06)$ \\
\hline Drug therapy & $1.37(0.91-2.06)$ & $1.86(1.01-3.42)$ & $0.98(0.57-1.71)$ & $1.49(0.65-3.41)$ \\
\hline $\operatorname{BMI}\left(\mathrm{kg} / \mathrm{m}^{2}\right)$ & $1.04(1.01-1.07)$ & $1.06(1.02-1.10)$ & $1.01(0.96-1.06)$ & $1.04(0.98-1.10)$ \\
\hline Waist circumference $(\mathrm{cm})$ & $1.03(1.02-1.04)$ & $1.03(1.02-1.05)$ & $1.02(1.00-1.04)$ & $1.04(1.02-1.06)$ \\
\hline Neck circumference $(\mathrm{cm})$ & $1.03(1.00-1.06)$ & $1.04(1.00-1.08)$ & $1.02(0.98-1.07)$ & $1.03(0.98-1.09)$ \\
\hline SBP $(\mathrm{mmHg})$ & $1.01(1.01-1.02)$ & $1.01(1.00-1.02)$ & $1.02(1.01-1.03)$ & $1.02(1.01-1.04)$ \\
\hline $\mathrm{DBP}(\mathrm{mmHg})$ & $0.99(0.98-1.01)$ & $1.00(0.98-1.01)$ & $0.99(0.98-1.01)$ & $0.99(0.96-1.01)$ \\
\hline $\mathrm{FPG}(\mathrm{mmol} / \mathrm{L})$ & $1.01(0.97-1.05)$ & $0.95(0.90-1.01)$ & $1.07(1.02-1.13)$ & $1.00(0.93-1.09)$ \\
\hline $\mathrm{Hpg}(\mathrm{mmol} / \mathrm{L})$ & $1.01(0.99-1.02)$ & $1.00(0.98-1.03)$ & $1.01(1.00-1.03)$ & $1.01(0.99-1.04)$ \\
\hline HbAlc (\%) & $1.08(1.01-1.14)$ & $1.03(0.94-1.12)$ & $1.14(1.05-1.24)$ & $1.16(1.05-1.29)$ \\
\hline $\mathrm{TG}(\mathrm{mmol} / \mathrm{L})$ & $1.04(0.97-1.11)$ & $1.01(0.91-1.11)$ & $1.07(0.97-1.18)$ & $0.99(0.85-1.15)$ \\
\hline $\mathrm{TC}(\mathrm{mmol} / \mathrm{L})$ & $1.08(1.00-1.16)$ & $1.03(0.92-1.14)$ & $1.14(1.04-1.25)$ & $0.95(0.79-1.13)$ \\
\hline HDL-cholesterol $(\mathrm{mmol} / \mathrm{L})$ & $0.88(0.67-1.15)$ & $0.60(0.40-0.89)$ & $1.26(0.92-1.74)$ & $0.47(0.25-0.89)$ \\
\hline LDL-cholesterol $(\mathrm{mmol} / \mathrm{L})$ & $1.08(0.95-1.22)$ & $1.16(0.99-1.36)$ & $0.97(0.80-1.18)$ & $0.97(0.77-1.23)$ \\
\hline Alanine aminotransferase $(10 \mathrm{U} / \mathrm{L})$ & $0.99(0.92-1.08)$ & $1.02(0.92-1.13)$ & $0.95(0.83-1.09)$ & $0.83(0.68-1.03)$ \\
\hline Blood urea nitrogen $(10 \mathrm{mmol} / \mathrm{L})$ & $0.98(0.89-1.08)$ & $0.92(0.69-1.22)$ & $1.01(0.92-1.11)$ & $1.02(0.91-1.14)$ \\
\hline Creatinine $(10 \mathrm{mmol} / \mathrm{L})$ & $1.03(0.99-1.06)$ & $1.01(0.96-1.06)$ & $1.05(1.00-1.11)$ & $1.05(0.99-1.12)$ \\
\hline Urine acid $(10 \mathrm{mmol} / \mathrm{l})$ & $1.02(1.01-1.03)$ & $1.02(1.00-1.03)$ & $1.02(1.00-1.03)$ & $1.02(1.00-1.04)$ \\
\hline I-FRS & $1.09(1.06-1.13)$ & $1.07(1.04-1.11)$ & $1.12(1.08-1.18)$ & $1.16(1.09-1.22)$ \\
\hline
\end{tabular}

Values in table are presented as the hazard ratio with the $95 \%$ confidence interval given in parenthesis

community hospitals and top tier hospitals could improve the current challenge of a lack of experts and managing a large population with T2DM and could also effectively improve the rate of achieving target control in patients with T2DM. This approach to individualized management employing a variety of treatment options may contribute to improved patientbased compliance and a reduction in the noncompliance rate.

Although excess mortality attributable to diabetes accounts for around 3 million deaths a year worldwide [11], little community-based research data are available on diabetes management in developing countries. This study is the first of the kind ever undertaken in China. The results of this study, which are similar to those of the Steno-2 study [12], provide novel data on the multifactor management of T2DM at a community level in China. Achievement of joint targets, including the achievement of targets for blood glucose, blood pressure, and lipid profiles, was $23.5 \%$, which may be explained by the comprehensive interventions that were applied. The American Association of Clinical Endocrinologists recommendations emphasize 
Table 5 Cox regression adjusted for age and clustering by clinic to evaluate the Framingham Risk Score as influenced by various cardiovascular events occurring in the Beijing Communities Diabetes Study

\begin{tabular}{|c|c|c|c|c|}
\hline \multirow[t]{2}{*}{ Endpoint events } & \multirow[t]{2}{*}{$P$} & \multirow[t]{2}{*}{ Hazard ratio } & \multicolumn{2}{|c|}{ 95\% Confidence interval for hazard ratio } \\
\hline & & & Lower & Upper \\
\hline \multicolumn{5}{|l|}{ CVD } \\
\hline I-FRS & 0.001 & & & \\
\hline $10-20 \%$ vs. $<10 \%$ & 0.003 & 3.89 & 1.60 & 9.47 \\
\hline$>20 \%$ vs. $<10 \%$ & 0.000 & 5.25 & 2.09 & 13.16 \\
\hline \multicolumn{5}{|l|}{ Cardiovascular } \\
\hline I-FRS & 0.150 & & & \\
\hline $10-20 \%$ vs. $<10 \%$ & 0.072 & 2.29 & 0.93 & 5.64 \\
\hline$>20 \%$ vs. $<10 \%$ & 0.052 & 2.60 & 0.99 & 6.79 \\
\hline \multicolumn{5}{|l|}{ Cerebrovascular } \\
\hline I-FRS & 0.005 & & & \\
\hline $10 \%-20 \%$ vs. $<10 \%$ & 0.041 & 7.82 & 1.08 & 56.35 \\
\hline$>20 \%$ vs. $<10 \%$ & 0.012 & 12.93 & 1.75 & 95.84 \\
\hline \multicolumn{5}{|c|}{ Cardiac and cerebral vascular death } \\
\hline I-FRS & 0.087 & & & \\
\hline $10 \%-20 \%$ vs. $<10 \%$ & 0.186 & 3.81 & 0.52 & 27.79 \\
\hline$>20 \%$ vs. $<10 \%$ & 0.088 & 5.81 & 0.77 & 43.90 \\
\hline
\end{tabular}

the importance of treating LDL-cholesterol) in individuals with diabetes [13]. The LDL-cholesterol target for lipid control was LDL-cholesterol $<2.6 \mathrm{mmol} / \mathrm{L}$ (with coronary heart disease $<1.8 \mathrm{mmol} / \mathrm{L}$ ).

However, during the process of management, the comprehensive compliance rate was observed to fluctuate greatly. Despite the presence of excellent doctors in the community setting, the joint target rate still fluctuated between 30 and $50 \%$. 31.5\% of patients achieved the joint target control more than three times in the Jinsong health center community. The ideal blood glucose control target was the most difficult to maintain during the long-term follow-up in relatively young patients with more social activities and obese in diabetes. Relaxation of lifestyle interventions may be the primary cause.
Some studies have found that approximately $75 \%$ of patients with T2DM are hypertensive [14]. In China, a report published in 2007 indicated that $34 \%$ of patients with T2DM had hypertension and that hypertension among those with diabetes was especially prevalent in northern China [15]. In the present study, $70.8 \%$ of the BCDS participants had hypertension. Although diabetes is itself a major cardiovascular risk factor, its presence increases the risk for other medical conditions, particularly hypertension [16]. In the United Kingdom Prospective Diabetes Study, tight blood pressure control in patients with hypertension and T2DM achieved a clinically important reduction in the risk of death and complications related to diabetes [17]. In Beijing communities, the patients in the medium and high FRS strata had higher SBP levels than those in the low FRS 
Table 6 Decrease in body mass index and the incidence of cardiovascular events occurring in the Beijing Communities Diabetes Study

\begin{tabular}{|c|c|c|c|c|}
\hline Endpoint events & $\begin{array}{l}\text { No decrease in BMI } \\
(n=2371 \text { patients })\end{array}$ & $\begin{array}{l}\text { Yes decrease in BMI } \\
(n=861 \text { patients })\end{array}$ & $\begin{array}{l}\text { Statistical } \\
\text { quantity }^{\mathrm{a}}\end{array}$ & $P$ \\
\hline CVD events & $254(10.71)$ & $89(10.34)$ & $0.09\left(\chi^{2}\right)$ & 0.759 \\
\hline Cardiovascular & $143(6.03)$ & $58(6.74)$ & $0.54\left(\chi^{2}\right)$ & 0.463 \\
\hline Myocardial infarction & $51(2.15)$ & $7(0.81)$ & $6.42\left(\chi^{2}\right)$ & 0.011 \\
\hline Unstable Angina pectoris & $16(0.67)$ & $12(1.39)$ & $3.80\left(\chi^{2}\right)$ & 0.051 \\
\hline Install cardiac pacemaker & $3(0.13)$ & $3(0.35)$ & $0.15(\mathrm{~F})$ & 0.196 \\
\hline Coronary stent implantation & $27(1.14)$ & $23(2.67)$ & $9.74\left(\chi^{2}\right)$ & 0.002 \\
\hline Coronary artery bypass grafting & $8(0.34)$ & $9(1.05)$ & $0.01(\mathrm{~F})$ & 0.024 \\
\hline Heart failure hospitalization & $19(0.80)$ & $2(0.23)$ & $3.17\left(\chi^{2}\right)$ & 0.075 \\
\hline Cerebrovascular & $111(4.68)$ & $31(3.60)$ & $1.76\left(\chi^{2}\right)$ & 0.185 \\
\hline Acute cerebral hemorrhage & $16(0.67)$ & $2(0.23)$ & $0.08(\mathrm{~F})$ & 0.183 \\
\hline Lacunar infarction & $26(1.10)$ & $4(0.46)$ & $2.74\left(\chi^{2}\right)$ & 0.098 \\
\hline $\begin{array}{l}\text { Acute cerebral infarction with limb } \\
\text { movement disorder }\end{array}$ & $55(2.32)$ & $14(1.63)$ & $1.45\left(\chi^{2}\right)$ & 0.228 \\
\hline Transient ischemic attack (TIA) & $12(0.51)$ & $10(1.16)$ & $4.01\left(\chi^{2}\right)$ & 0.045 \\
\hline All-cause death & $185(7.80)$ & $3(0.35)$ & $64.06\left(\chi^{2}\right)$ & $<0.001$ \\
\hline Cardiac and cerebral vascular death & $89(3.75)$ & $1(0.12)$ & $30.87\left(\chi^{2}\right)$ & $<0.001$ \\
\hline Cardiac death & $62(2.61)$ & $1(0.12)$ & $20.63\left(\chi^{2}\right.$ & $<0.001$ \\
\hline Cerebral death & $27(1.14)$ & $0(0.00)$ & $9.89\left(\chi^{2}\right)$ & 0.002 \\
\hline
\end{tabular}

Values in table are presented as the number of patients with the percentage in parenthesis

${ }^{a}$ F, Fisher's exact test; $\chi^{2}$, Chi-square test

stratum. Thus, the need for multirisk factor control and effective community-based management for patients with T2DM and hypertension should be emphasized.

Several follow-up studies have suggested that weight change is a mechanism for reducing the incidence of diabetes [18]. The BCDS-2 study focused on the clinical characteristics of diabetic patients with metabolic syndrome and its components living in urban communities in Beijing in 2018 [19]. Visceral adipose tissue is recognized as a unique, pathogenic fat depot that confers a metabolic risk above and beyond standard anthropometric measures, such as BMI and waist circumference [20]. As an index for upper-body subcutaneous adipose tissue distribution, neck circumference seems to be a suitable parameter for use in a clinical setting as a strong indicator of central obesity and metabolic abnormalities in persons with T2DM, and this parameter has been evaluated in relation to cardiovascular risk factors $[10,21]$. In our factor analyses performed to explore the variables contributing to the incidence of CVD events, we considered such variables as BMI and neck circumference as potential confounding factors. In the BCDS, a decrease in the BMI was found to be closely related to all-cause death and cardiovascular events, especially in myocardial infarction, coronary stent implantation and 
coronary artery bypass grafting. Despite the increasing number of studies on neck circumference and cardiovascular events, this correlation is important as it can shed some light on the pathogenesis of CVD and merits further long-term research.

The importance of individualized risk assessment is receiving ever more attention. Many studies have shown that the use of different cardiovascular risk assessment tools for the same individual can result in the risk of CVD over a 10 -year period being very different or the prediction even placing the individual in a different risk stratification. Tools are valuable to clinics, where it is important to define the risks for a specific individual and then develop interventions in individuals. Therefore, the availability of more accurate risk predicts tools will facilitate more effective intervention. At present, there is a lack of comprehensive, individualized and more economical CVD risk assessment tools in China. Therefore, it is necessary to discuss the CVD risk assessment tools suitable for Chinese patients with diabetes.

As complications related to CVD are still a very important health problem, the FRS has become a widely used method to predict the occurrence of CVD over a 10-year period, and its usefulness in this regard has been demonstrated [6]. Studies have also shown that the higher the risk for developing CVD, the greater the efficacy of the intervention that is needed. Several primary prevention trials have demonstrated a significant effect of interventions on cardiovascular events in patients with lower risk scores [22].

In our study, patients in medium and high FRS strata were more likely to be older and have a longer duration of diabetes and higher blood pressure than those in the low FRS stratum. At the end of the 10-years of follow-up, the I-FRS was significantly lower in the low, medium and high FRS strata $(P<0.001)$. In addition, at baseline and after 10-years of follow-up, the post-intervention I-FRS was significantly lower than the baseline score in the HTN group and CVD group ( $P<0.01$ for each); in contrast, at the end of the 10-years of follow-up, there was no significant difference in the I-FRS of the DM group when compared with baseline data. Thus, it is likely that a multifactorial intervention for CVD risk factors will have a positive, long-term effect on the I-FRS for patients with T2DM in the community, especially those in the high FRS stratum. It appears that paying more attention to patients with T2DM with hypertension or CVD disease will be more effective during the multifactorial intervention. Some indicators for calculating the FRS can be changed, and some cannot be changed, such as age. During the multifactor intervention, metabolic indicators can be changed by treatment and, therefore, the prevention of these indicators will have important value for CVD prevention in practice.

Despite the effects of multifactorial interventions on the I-FRS, we detected an increased incidence of cardiovascular events during the 10-year follow-up period. The 10-year HRs for CVD events in diabetic patients with an I-FRS score $>20 \%$ was 12.5 -fold higher than that of patients with an I-FRS score $<10 \%$. The cumulative CVD event rate was significantly higher among patients in the medium and high FRS strata among those in the low FRS strata. The 10-year HRs for death caused by CVD events in diabetic patients with FRS score $>20 \%$ was 2.91-fold higher than that of patients with an I-FRS score $<10 \%$. The observation that CVD events seem to have a reduced incidence in people who had received specific interventions during the follow-up period is encouraging.

This study was a prospective one, and the dataset used for the analyses has considerable strength. A large, ethnically-diverse community population was enrolled, detailed clinical and metabolic characterization of the cohort was achieved, and important data were centralized and tested in a central laboratory.

\section{Study Limitations}

As the study was based on a selected population, it has some limitations. Patients with a non-first CVD were mainly treated in accordance with national guidelines for CVD, which resulted in lifestyle and body weight changes. However, if the associations that we observed between neck circumference and CVD are confirmed in future 
studies in first-time CVD patients, a higher neck circumference could serve as a risk stratification tool for CVD in patients with diabetes mellitus.

This study was carried out only in the urban area of Beijing, and the results will be supported in the furture by a nationwide multi-center prospective clinical study. Nutritional measures can play a very important role in development of outcomes investigated in this study. However, these measures were not considered in analysis.

\section{CONCLUSION}

In summary, increased blood pressure, abnormal lipid profiles, and elevated plasma glucose concentrations were highly significantly associated with CVD in the study population. Moreover, it was evident that the I-FRS was more suitable in patients with T2DM and was found to be a useful tool for estimating the beneficial effects of multifactorial treatments in the DM, HTN, and CVD groups. Especially for diabetic patients with a risk score of $>20 \%$, multifactorial treatment could be effective in improving the I-FRS. Thus, multifactorial interventions for CVD risk factors in the community are important to reduce the incidence of endpoint events in Chinese diabetic patients with hypertension or CVD. Certain groups of patients may benefit from I-FRS estimate as a tool to aid decision-making regarding overall risk reduction. Hence, we suggest the use of the I-FRS to stratify patients' CVD risk when analyzing the efficacy of management at regular intervals. Aggressive risk reduction should be focused on those individuals who had a high I-FRS and to achieving individualized risk assessment and improving the effectiveness and price ratio of the intervention.

\section{ACKNOWLEDGEMENTS}

We thank the participants and general practitioners who took part in the study. Zong-xue $\mathrm{Lu}$, Shu-xian Ma, Shu-qin Yang, and Wei Bai are thanked as members of the Supervisory Committee.
Funding. This project is supported by a Grant of Special Scientific Research on Capital Health Development (2016-1-2057, 2016-22054), Beijing Municipal Science \&Technology Commission (Z151100004015021), BRIDGES Grant from the International Diabetes Federation. The Rapid Service Fee was funded by the authors.

Authorship. All named authors meet the International Committee of Medical Journal Editors (ICMJE) criteria for authorship for this article, take responsibility for the integrity of the work as a whole, and have given their approval for this version to be published.

Authorship Contributions. SY was principal investigator and was responsible for the study conception and design in the BCDS study. RRH was responsible for the study conception and design in the BCDS study. X-LZ drafted the manuscript and contributed to the collection and standardization of the data. WG conducted the principal statistical analysis, data analysis, and interpretation. All authors contributed to the writing of this paper and approved the final version.

List of Investigators. The full list of the BCDS Study Group Investigators can be found in the Electronic Supplementary Material.

Disclosures. Xue-Lian Zhang, Gang Wan, Ming-Xia Yuan, Guang-Ran Yang, Han-Jing Fu, Liang-Xiang Zhu, Rong-Rong Xie, Yu-Jie Lv, Jian-Dong Zhang, Yu-Ling Li, Qin-Fang Dai, Yu Ji, Rury R. Holman and Shen-Yuan Yuan have nothing to disclose.

Compliance with Ethics Guidelines. The study was conducted in accordance with the Helsinki Declaration of 1964, as revised in 2013 and was approved by the Medical Ethics Committee of Beijing Tongren Hospital. Informed consent was obtained from all patients for being included in the study.

Data Availability. The datasets during and/ or analyzed during the current study are 
available from the corresponding author on reasonable request.

Open Access. This article is licensed under a Creative Commons Attribution-NonCommercial 4.0 International License, which permits any non-commercial use, sharing, adaptation, distribution and reproduction in any medium or format, as long as you give appropriate credit to the original author(s) and the source, provide a link to the Creative Commons licence, and indicate if changes were made. The images or other third party material in this article are included in the article's Creative Commons licence, unless indicated otherwise in a credit line to the material. If material is not included in the article's Creative Commons licence and your intended use is not permitted by statutory regulation or exceeds the permitted use, you will need to obtain permission directly from the copyright holder. To view a copy of this licence, visit http:// creativecommons.org/licenses/by-nc/4.0/.

\section{REFERENCES}

1. Stern MP, Haffner SM. Prospective assessment of metabolic control in diabetes mellitus: the complications question. JAMA. 1988;260(19):2896-7.

2. Chen L, Magliano DJ, Zimmet PZ. The worldwide epidemiology of type 2 diabetes mellitus-present and future perspectives. Nat Rev Endocrinol. 2011;8(4):228-36. https://doi.org/10.1038/nrendo. 2011.183.

3. American Diabetes Association: Diabetes statistics 2011. Updated 26 Jan 2011. http://www.diabetes. org/diabetes-basics/diabetes-statistics. Accessed 7 Oct 2014

4. UK Prospective Diabetes Study Group. Tight blood pressure control and risk of macrovascular and microvascular complications in type 2 diabetes: UKPDS 38. BMJ. 1998;317:703-13.

5. Yang W, Lu J, Weng J, et al. Prevalence of diabetes among men and women in China. N Engl J Med. 2010;362(12):1090-101. https://doi.org/10.1056/ nejmoa0908292.

6. Ryoo JH, Cho SH, Kim SW. Prediction of risk factors for coronary heart disease using Framingham risk score in Korean men. PLoS One. 2012;7:e45030. https://doi.org/10.1371/journal.pone.0045030.

7. Minhas JS, Patel P, Gupta PK. Limitations of a Cardiac Risk (QRISK2) Calculator in Patients with High Density Lipoprotein (HDL). High Blood Press Cardiovasc Prev. 2016;23(1):47-50. https://doi.org/10. 1007/s40292-016-0131-X.

8. $\mathrm{Xu} \mathrm{J}, \mathrm{Wei} \mathrm{WB}$, Yuan MX, et al. Prevalence and risk factors for diabetic retinopathy: the Beijing Communities Diabetes Study 6. Retina. 2012;32:322-9. https://doi.org/10.1097/IAE.0b013e31821c4252.

9. Wilson PW, D'Agostino RB, Levy D, et al. Prediction of coronary heart disease using risk factor categories. Circulation. 1998;97:1837-47.

10. Yang GR, Yuan SY, Fu HJ, et al. Beijing Community Diabetes Study Group. Beijing Community Diabetes Study Group: Neck circumference positively related with central obesity, overweight, and metabolic syndrome in Chinese subjects with type 2 diabetes: Beijing Community Diabetes Study 4. Diabetes Care. 2010;33:2465-7. https://doi.org/10. 2337/dc10-0798.

11. Jafar TH, Haaland BA, Rahman A, et al. Non-communicable diseases and injuries in Pakistan: strategic priorities. Lancet. 2013;381:2281-90. https:// doi.org/10.1016/S0140-6736(13)60646-7.

12. Gaede P, Lund-Andersen H, Parving HH, Pedersen O. Effect of a multifactorial intervention on mortality in type 2 diabetes. N Engl J Med. 2008;358: 580-91. https://doi.org/10.1056/NEJMoa0706245.

13. Jellinger PS, Handelsman Y, Rosenblit PD, et al. American Association of Clinical Endocrinologists and American College of Endocrinology Guidelines for the Management of Dyslipidemia and Prevention of Cardiovascular Disease. Endocr Pract. 2017;23[Suppl 2]:1-87. https://doi.org/10.4158/ EP171764.APPGL.

14. Cooper-DeHoff RM, Pacanowski MA, Pepine CJ. Cardiovascular therapies and associated glucose homeostasis: implications across the dysglycemia continuum. J Am Coll Cardiol. 2009;53[5 Suppl]: S28-34. https://doi.org/10.1016/j.jacc.2008.10.037.

15. Qi W, Pan C, Lin S. A survey of factors influencing prognosis and control rate for patients with hypertension in mainland China. Chin J Cardiol. 2007;35:457-60.

16. Gillespie EL, White CM, Kardas M, et al. The impact of ACE inhibitors or angiotensin II type 1 receptor blockers on the development of new-onset type 2 diabetes. Diabetes Care. 2005;28:2261-6. 
17. UK Prospective Diabetes Study Group. Tight blood pressure control and risk of macrovascular and microvascular complications in type 2 diabetes: UKPDS 38. BMJ, 1998, 317: 703-13 ; 1999, 318: 29. PMID:9732337.

18. Penn L, White M, Lindström J, et al. Importance of weight loss maintenance and risk prediction in the prevention of type 2 diabetes: analysis of European Diabetes Prevention Study RCT. PLoS One. 2013;8: e57143. https://doi.org/10.1371/journal.pone. 0057143.

19. Fu H, Yuan S, Wan G, et al. Clinical characteristics of diabetic patients with metabolic syndrome and its components at 15 urban communities in Beijing. Chin J Gen Pract. 2011;10:390-3.
20. Fox CS, Massaro JM, Hoffmann U, et al. Abdominal visceral and subcutaneous adipose tissue compartments: association with metabolic risk factors in the Framingham Heart Study. Circulation. 2007;116: 39-48. https://doi.org/10.1161/CIRCULATIONAHA. 106.675355 .

21. Preis SR, Massaro JM, Hoffmann U, et al. Neck circumference as a novel measure of cardiometabolic risk: the Framingham Heart study. J Clin Endocrinol Metab. 2010;95:3701-10. https://doi.org/10. 1210/jc.2009-1779.

22. Downs JR, Clearfield M, Weis S, et al. Primary prevention of acute coronary events with lovastatin in men and women with average cholesterol levels: results of AFCAPS/TexCAPS Air Force/Texas Coronary Atherosclerosis Prevention Study. JAMA. 1998;279:1615-22. 